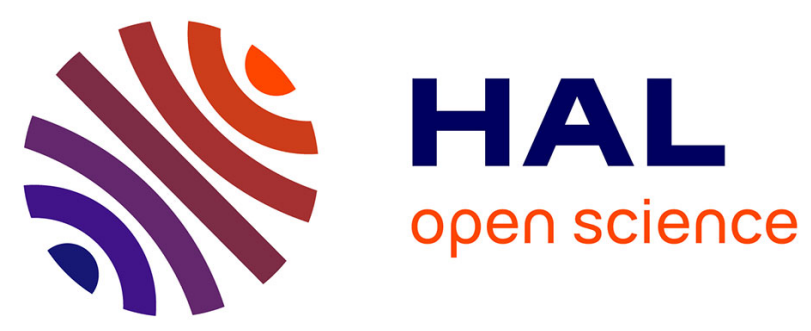

\title{
PI Passivity-based Control of Modular Multilevel Converters for Multi-Terminal HVDC Systems
}

Gilbert Bergna-Diaz, Daniele Zonetti, Santiago Sanchez, Elisabetta Tedeschi, Romeo Ortega

\section{- To cite this version:}

Gilbert Bergna-Diaz, Daniele Zonetti, Santiago Sanchez, Elisabetta Tedeschi, Romeo Ortega. PI Passivity-based Control of Modular Multilevel Converters for Multi-Terminal HVDC Systems. IEEE 18th Workshop on Control and Modeling for Power Electronics (COMPEL), Jul 2017, Stanford, United States. 10.1109/COMPEL.2017.8013329 . hal-02378404

\section{HAL Id: hal-02378404 https://hal.science/hal-02378404}

Submitted on 26 Jun 2020

HAL is a multi-disciplinary open access archive for the deposit and dissemination of scientific research documents, whether they are published or not. The documents may come from teaching and research institutions in France or abroad, or from public or private research centers.
L'archive ouverte pluridisciplinaire HAL, est destinée au dépôt et à la diffusion de documents scientifiques de niveau recherche, publiés ou non, émanant des établissements d'enseignement et de recherche français ou étrangers, des laboratoires publics ou privés. 


\title{
PI Passivity-based Control of Modular Multilevel Converters for Multi-Terminal HVDC Systems
}

\author{
Gilbert Bergna-Diaz*, Daniele Zonetti ${ }^{\dagger}$, Santiago Sanchez*, Elisabetta Tedeschi* and Romeo Ortega ${ }^{\ddagger}$ \\ * Norwegian Univ. of Science and Technology - Dept. Electric Power Engineering - Trondheim, Norway. \\ ${ }^{\dagger}$ IMDEA Energy Institute - Electrical Systems Unit - Madrid, Spain. \\ ¥ Laboratoire des Signeaux et Systèmes/CNRS/Supélec - Gif sur Yvette, France.
}

\begin{abstract}
In this work, a decentralized PI passivity-based controller (PI-PBC) is applied to the Modular Multilevel Converters (MMCs) to ensure global asymptotic stability of a multiterminal MT-HVDC system. Since continuous MMC state-space models naturally have time-periodic steady-state solutions, a first step towards the derivation of the controller is the formulation of an equivalent model characterized by constant steady-state solutions, obtained via a multi-frequency orthogonal coordinates transformation. For the design of the controller, the overall system is represented in an appropriate port-Hamiltonian formulation, which allows the derivation of the stabilizing control law using passivity-based arguments. The results are validated on a threeterminal simulation benchmark.
\end{abstract}

\section{INTRODUCTION}

The integration of large volumes of renewable energy systems into the existing power grid constitutes one of the main pillars towards the much needed low-carbon economy. However, due to their intermittent nature and uneven geographical distribution, major developments on the grid infrastructure are likely required [1]. One possibility is to conceive new architectures that will take place at the boundary of the existing infrastructure, both at the low- and high-voltage levels. At the high-voltage level, a transmission system spanning the whole planet and connecting most of the large power plants in the world has been proposed, the so-called global grid or supergrid concept [2]. In this scheme it would be possible to integrate remotely located, high potential, aggregated renewable energy resources providing sustainable energy to the main grids by means of multi-terminal high-voltage direct-current (MTHVDC) transmission systems [3].

MT-HVDC grids are expected to be multi-vendor by nature, thus posing the problem of interoperability of their components, in particular of the power converters and the correspondent controllers [1]. Although the concept of interoperability has several valid interpretations, it is widely acknowledged that a key role is played by the analysis of stability and performance properties [4]. Unfortunately, two practical aspects complicate the stability analysis. First, since the supergrid is expected to grow organically and plug \& play operation of the components is likely required, system structural changes may invalidate the stability results obtained for the original architecture. Second, the controllers implemented by the vendors are usually subjected to confidentiality agreements. In this context, it is necessary to establish well-defined grid codes - to be complied locally by the vendors - that guarantee an appropriate operation of the grid. These codes must ensure that the stability of the overall system is preserved, in presence of structural changes, and with no need of sharing information on the protected control strategies.

A possible approach for the assessment of stability for large-scale, nonlinear, interconnected systems is based on passivity theory [5]. In particular, for the case of MT-HVDC system, it was shown in [6], [7] that - using passivity argumentsappropriate, decentralized PI controllers, can be designed to guarantee stability of the closed-loop system. These works considered MT-HVDC based on two-level voltage source converters (2L-VSCs).

The present paper aims at extending these results for the MT-HVDC systems based on Modular Multilevel Converters (MMCs), which are a recent, emerging topology of power converters [8]. Compared to the 2L-VSC, the MMC is characterized by additional internal dynamics, which are related to the circulating currents and the internal capacitor voltages of the upper and lower arms of each phase [9], [10]. The main contribution of this paper is inspired by the results originally presented in [11] for a $2 \mathrm{~L}-\mathrm{VSC}$ in single-terminal configuration-and then generalized in [6], [7] for HVDC systems. Nevertheless, the application of such methodology to an MMC is not straightforward for two fundamental reasons. First, conventional coordinate transformations fail to map the desired steady-state behavior of the system, which consist of periodic solutions, to a fixed equilibrium point, thus stimying the formulation of the control problem as a pure regulation problem. Second, the results presented in [11] require the system to be represented in a suitable port-Hamiltonian form that is not obvious, due to the complexity of the system. To overcome these problems, an equivalent averaged formulation that has constant steady-state solutions is obtained (along the lines of [12], [13]). Then, the model is represented in port-Hamiltonian form [14], following the procedure used in [15] for general power converters. Based on the obtained representation, the results of [11] are then applied to a single MMC and extended to the case of a general MMC-based MTHVDC transmission system with radial topology. The stability properties of the resulting controllers are validated via timedomain simulations on a three-terminal configuration.

The remainder of the paper is organized as follows: the derivation of the averaged model of the MMC with constant steady-state is presented in Section II. After a brief recall of the results of [11], the PI-PBC method is applied to the MMC model in single-terminal HVDC configuration in Section IIIand extended to the multi-terminal case in Section IV. Simulation results illustrating the performance of the proposed controller are provided in Section V. We wrap-up the paper 


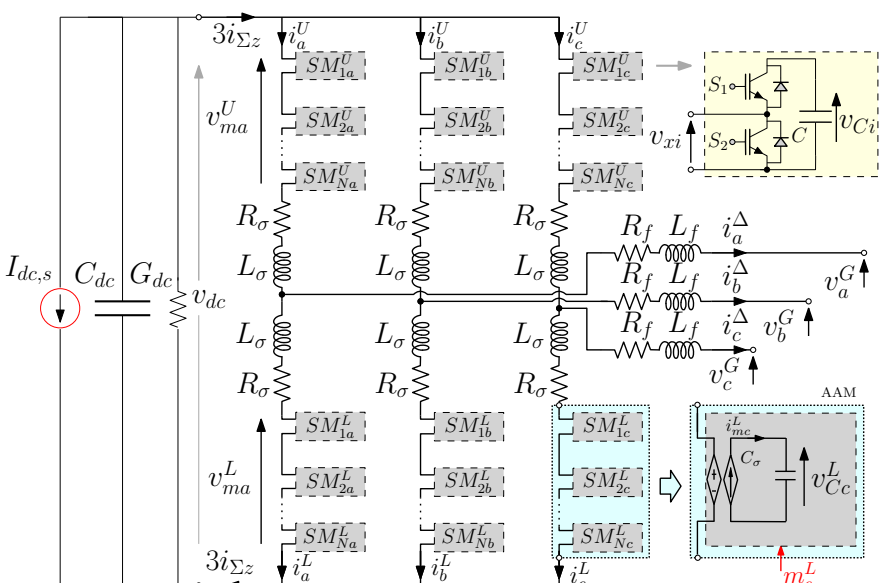

Figure 1: Topology of the modular multilevel converter under consideration

with conclusions and guidelines for future research in Section VI.

\section{MOdeling OF MODUlaR MULTILEVEL CONVERTERS}

\section{A. Arm Averaged Model in $\Sigma-\Delta$ coordinates}

A single MMC with three upper and three lower arms in half bridge configuration is considered, the topology of which is represented in full-detail in Fig. 1. Both upper and lower arms are constituted by an arm inductor-characterized by an inductance $L_{\sigma}$ and resistance $R_{\sigma}$-connected in series with $N$ sub-modules (SMs). Each SM includes two IGBTs, their respective free wheeling diode and an equivalent capacitor. Moreover, an $R L$ element is usually added on the ac side, in order to take into account the leakage inductance of the transformer and additional inductive filters.

Due to the complexity of the system, suitable, simplified models are usually employed in literature, based on the following assumptions—see [10], [16], [17] for more details.

A1. Switching dynamics can be neglected.

A2. All SMs voltage signals in the same arm are identical.

A3. Balanced operation of the phases.

Assumption A1 is justified by the very fast operation of the switches, compared to the time-scale at which the other electrical variables evolve. Assumption A2 is justified for MMC characterized by a large number of levels, see [18], and whenever a very fast arm capacitor balancing algorithm is applied. Assumption A3 is justified by appropriate design of the system components. Under these assumptions, the switched model of the series-connected SMs can be approximated by their average, which leads to the following average model for the upper and lower arms $j \in\{U, L\}$ :

$$
\begin{aligned}
C_{\sigma} \dot{v}_{C}^{j} & =m^{j} \circ i^{j} \\
L_{\sigma} \dot{i}^{j} & =-R_{\sigma} i^{j}-m^{j} \circ v_{C}^{j}+v_{\sigma}^{j},
\end{aligned}
$$

where the symbol $\circ$ denotes the Hadamard product, i.e. the element-wise multiplication between vectors, and with: state vector composed by the three-phase arm voltages $v_{C}^{j} \in \mathbb{R}^{3}$ and currents $i^{j} \in \mathbb{R}^{3}$; control vector the three-phase insertion indices $m^{j} \in \mathbb{R}^{3}$; input variable the three-phase voltage $v_{\sigma}^{j} \in \mathbb{R}^{3}$; scalar parameters $C_{\sigma}, L_{\sigma}, R_{\sigma} \in \mathbb{R}_{+}$corresponding to the arm equivalent capacitance, inductance and resistance respectively.

In order to interconnect the arms with the $R L$ element preceding the grid interface, the model (1) can be transformed in more suitable $\Sigma-\Delta$ coordinates, which are defined as follows:

$$
\begin{aligned}
v_{C}^{\Sigma} & :=v_{C}^{U}+v_{C}^{L}, \quad v_{C}^{\Delta}:=v_{C}^{U}-v_{C}^{L} \\
i^{\Sigma} & :=\frac{1}{2}\left(i^{U}+i^{L}\right), \quad i^{\Delta}:=i^{U}-i^{L} \\
m^{\Sigma} & :=m^{U}+m^{L}, \quad m^{\Delta}:=m^{U}-m^{L},
\end{aligned}
$$

where currents $i^{\Sigma}$ and $i^{\Delta}$ have clear physical meanings, i.e. they correspond to the three-phase circulating current and the three-phase current flowing into the grid through the $R L$ element respectively. After some manipulations, the arms dynamics in $\Sigma-\Delta$ coordinates can be combined with the dynamics of the $R L$ element, thus leading to:

$$
\begin{aligned}
C_{\sigma} \dot{v}_{C}^{\Sigma} & =m^{\Sigma} \circ i^{\Sigma}+\frac{1}{4} m^{\Delta} \circ i^{\Delta} ; \\
C_{\sigma} \dot{v}_{C}^{\Delta} & =m^{\Delta} \circ i^{\Sigma}+\frac{1}{4} m^{\Sigma} \circ i^{\Delta} ; \\
L_{\sigma} i^{\Sigma} & =\frac{1}{2} 1_{3} v_{d c}-R_{\sigma} i^{\Sigma}-\frac{1}{4}\left(m^{\Sigma} \circ v_{C}^{\Sigma}+m^{\Delta} \circ v_{C}^{\Delta}\right) ; \\
L_{\delta} \dot{i}^{\Delta} & =-R_{\delta} i^{\Delta}-\frac{1}{4}\left(m^{\Sigma} \circ v_{C}^{\Delta}+m^{\Delta} \circ v_{C}^{\Sigma}\right)-v_{G}^{\Delta} ;
\end{aligned}
$$

where $v_{G}^{\Delta} \in \mathbb{R}^{3}$ is the three-phase grid voltage, oscillating at the fundamental frequency $\omega \in \mathbb{R}_{+}$and where the equivalent ac inductance $L_{\delta}=L_{\sigma} / 2+L_{f}$ and resistance $R_{\delta}=R_{\sigma} / 2+$ $R_{f}$ have been further introduced. The obtained model (2) is referred in the sequel as the Arm Averaged Model (AAM) of the MMC.

\section{B. Steady-state analysis}

A fundamental step towards the analysis and the control design is the identification of the steady-states of interest for the correct and safe operation of an MMC. The standard requirements are the following-see [8], [19] for a precise justification:

R1. The ac grid current must be, at steady-state, a periodic three-phase sinusoidal signal at the fundamental frequency $\omega$, i.e.

$$
i_{\mathrm{ss}}^{\Delta}=I_{\mathrm{ss}}^{\Delta} \overline{\sin }\left(\omega t+\varphi_{\mathrm{ss}}^{\Delta}\right), \quad I_{\mathrm{ss}}^{\Delta} \in \mathbb{R}, \varphi_{\mathrm{ss}}^{\Delta} \in \mathbb{S} ;
$$

with $\overline{\sin }(\cdot), \overline{\cos }(\cdot) \in \mathbb{R}^{3}$ denoting three-phase balanced signals.

R2. The upper arm insertion indexes $m_{\mathrm{ss}}^{U}$ must be phaseshifted approximately of 180 degrees with respect to the lower arm insertion index $m_{\mathrm{ss}}^{L}$, resulting in

$$
m_{\mathrm{ss}}^{\Sigma} \approx 1_{3}, \quad m_{\mathrm{ss}}^{\Delta} \approx M_{\mathrm{ss}} \overline{\sin }(\omega t) ;
$$

R3. the sum of the circulating current in all phases must be a dc signal, i.e.

$$
1_{3}^{\top} i_{\mathrm{ss}}^{\Sigma}=\text { const }
$$

R4. the sum of all arm capacitor voltages must be a dc 
signal, i.e.

$$
1_{3}^{\top} v_{C, \mathrm{ss}}^{\Sigma}=\text { const. }
$$

These requirements complicate the control design, since the steady-state of interest does not coincide with a fixed equilibrium point. In order to overcome this problem, a possibility is to employ an additional transformation of coordinates so that the resulting system is characterized by time-invariant steadystates. In order to determine the appropriate transformation to be employed, an analysis of the oscillations of the steady-states arising from (2) has been carried-out in [12]-[14], further validated by extensive simulations. In particular, under the above mentioned requirements, it was shown that the following approximations hold at steady-state conditions:

- $\Delta$-variables are the sum of a signal oscillating at the fundamental frequency $\omega$ with a signal oscillating at frequency $3 \omega$

- $\quad \Sigma$-variables are the sum of a dc signal with a signal oscillating at frequency $-2 \omega$.

Notice that special cases of such a classification are the grid current $i^{\Delta}$, which only oscillates at the fundamental frequency as established by the Requirement $\mathrm{R} 1$, and the circulating current $i^{\Sigma}$, that might consists of a simple dc signal, in the case that a Circulating Current Suppression Controller is adopted, see for example [20].

Remark 1. In [12], [13] it was demonstrated that, at steadysate, $\Sigma$ - and $\Delta$-variables are further characterized by additional higher order harmonics. Nevertheless, extensive time-domain simulations suggested that these are not especially relevant in the system dynamics and therefore can be neglected.

\section{A multi-frequency coordinates transformation}

The classification presented in the previous section is useful to determine a suitable, multi-frequency coordinates transformation that maps the oscillating steady-states of interest to constant quantities. This approach, originally presented in [12], [13], is summarized in Fig. 2 and is based on an appropriate combination of Park and rotational transformations.

Recall that a Park transformation of a general three-phase signal $x=\operatorname{col}\left(x_{a}, x_{b}, x_{c}\right) \in \mathbb{R}^{3}$ with tranformation angle $\phi(t)=\omega_{0} t \in \mathbb{R}$, is defined by:

$$
x_{d q z}:=\frac{2}{3}\left[\begin{array}{c}
\overline{\cos }\left(\omega_{0} t\right)^{\top} \\
\sin \left(\omega_{0} t\right)^{\top} \\
\frac{1}{2} 1_{3}^{\top}
\end{array}\right]\left[\begin{array}{l}
x_{a} \\
x_{b} \\
x_{c}
\end{array}\right]=P_{\omega_{0}} x,
$$

with $x_{d q z}=\operatorname{col}\left(x_{d}, x_{q}, x_{z}\right) \in \mathbb{R}^{3}$. Let then $x^{\Delta} \in \mathbb{R}^{3}$, $x^{\Sigma} \in \mathbb{R}^{3}$, be general three-phase signals in $\Delta$-, $\Sigma$-coordinates respectively, and define the following transformations:

$$
x_{d q z}^{\Sigma}:=P_{-2 \omega} x^{\Sigma}, \quad x_{d q z}^{\Delta}:=P_{\omega} x^{\Delta} .
$$

An immediate consequence of using these transformations and of the classification introduced in Section II-B, is that in steady-state conditions, $\Sigma$-variables are mapped to a constant vector $x_{\mathrm{ss}, d q z}^{\Sigma}$. By contrast, $\Delta$-variables are mapped to a vector

$$
x_{\mathrm{ss}, d q z}^{\Delta}=\operatorname{col}\left(x_{d, \mathrm{ss}}^{\Delta}, x_{q, \mathrm{ss}}^{\Delta}, x_{z, \mathrm{ss}}^{\Delta}\right) \in \mathbb{R}^{3},
$$

where $x_{d, \mathrm{ss}}^{\Delta}, x_{q, \mathrm{ss}}^{\Delta}$ are constant quantities, while the correspondent zero-component $x_{z, \mathrm{ss}}^{\Delta}$ still oscillates at frequency

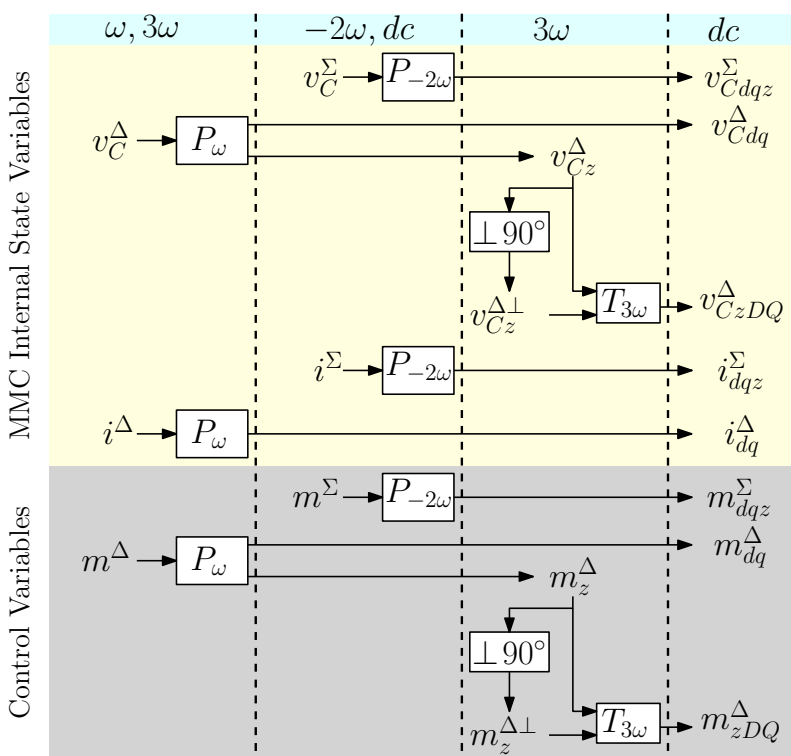

Figure 2: Multi-frequency coordinates transformation.

$3 \omega$. It is thus possible to rewrite the AAM model (2) in new coordinates-see [12], [13] for a detailed derivation-as follows:

$$
\begin{aligned}
C_{\sigma} \dot{v}_{C d q z}^{\Sigma} & =\mathbb{J}_{3} C_{\sigma} 2 \omega v_{C d q z}^{\Sigma}+I_{d q z}^{\Sigma} \\
C_{\sigma} \dot{v}_{C d q z}^{\Delta} & =\mathbb{J}_{3} C_{\sigma} \omega v_{C d q z}^{\Delta}+I_{d q z}^{\Delta} \\
L_{\sigma} \dot{i}_{d q z}^{\Sigma} & =\frac{1}{2} e_{3} v_{d c}+\left(\mathbb{J}_{3} L_{\sigma} 2 \omega-R_{\sigma}\right) i_{d q z}^{\Sigma}-V_{d q z}^{\Sigma} \\
L_{\delta} \dot{i}_{d q z}^{\Delta} & =\left(\mathbb{J}_{3} L_{\delta} \omega-R_{\delta}\right) i_{d q z}^{\Delta}+V_{d q z}^{\Delta}-v_{G d q z}^{\Delta}
\end{aligned}
$$

with

$$
e_{3}:=\left[\begin{array}{l}
0 \\
0 \\
1
\end{array}\right] \in \mathbb{R}^{3}, \quad \mathbb{J}_{3}:=\left[\begin{array}{ccc}
0 & 1 & 0 \\
-1 & 0 & 0 \\
0 & 0 & 0
\end{array}\right] \in \mathbb{R}^{3 \times 3}
$$

and the following definitions:

$$
\begin{aligned}
I_{d q z}^{\Sigma} & :=P_{-2 \omega}\left[P_{-2 \omega}^{-1} m_{d q z}^{\Sigma} \circ P_{-2 \omega}^{-1} i_{d q z}^{\Sigma}+P_{\omega}^{-1} m_{d q z}^{\Delta} \circ \frac{1}{2} P_{\omega}^{-1} i_{d q z}^{\Delta}\right] \\
I_{d q z}^{\Delta}: & =P_{\omega}\left[P_{\omega}^{-1} m_{d q z}^{\Delta} \circ P_{-2 \omega}^{-1} i_{d q z}^{\Sigma}+P_{-2 \omega}^{-1} m_{d q z}^{\Sigma} \circ \frac{1}{2} P_{\omega}^{-1} i_{d q z}^{\Delta}\right] \\
V_{d q z}^{\Sigma}: & =\frac{1}{4} P_{-2 \omega}\left[P_{-2 \omega}^{-1} m_{d q z}^{\Sigma} \circ P_{-2 \omega}^{-1} v_{C d q z}^{\Sigma}+P_{\omega}^{-1} m_{d q z}^{\Delta} \circ P_{\omega}^{-1} v_{C d q z}^{\Delta}\right] \\
V_{d q z}^{\Delta}: & =-\frac{1}{4} P_{\omega}\left[P_{\omega}^{-1} m_{d q z}^{\Delta} \circ P_{-2 \omega}^{-1} v_{C d q z}^{\Sigma}+P_{-2 \omega}^{-1} m_{d q z}^{\Sigma} \circ P_{\omega}^{-1} v_{C d q z}^{\Delta}\right]
\end{aligned}
$$

with $I_{d q z}^{\Sigma}, I_{d q z}^{\Delta}$ and $V_{d q z}^{\Sigma}, V_{d q z}^{\Delta}$ corresponding to the threephase modulated current and voltages respectively. It is immediate to see that, due to the Park transformations employed, these variables depend on $\omega$, which imply the generation of harmonics at steady-state. A more accurate analysis of these terms shows that, in accordance with the steady-state analysis previously performed, harmonics are characterized by frequencies $3 \omega$ and $6 \omega$. Although sixth-order harmonics are negligible, care must be exercised in handling third-order harmonics. Hence, as illustrated in Fig. 2, a further, rotational transformation is employed to map these harmonics to constant values. This type of transformation is usually adopted for the description of single-phase ac systems in a stationary reference frame [21] and employs an auxiliary, virtual variable $x^{\perp} \in \mathbb{R}$, 
shifted of 90 degrees from the original signal $x \in \mathbb{R}$. More precisely, it is defined as:

$$
x_{D Q}:=\left[\begin{array}{rr}
\cos \left(\omega_{0} t\right) & \sin \left(\omega_{0} t\right) \\
-\sin \left(\omega_{0} t\right) & \cos \left(\omega_{0} t\right)
\end{array}\right]\left[\begin{array}{l}
x \\
x^{\perp}
\end{array}\right]=T_{\omega_{0}} x,
$$

with $x_{D Q}=\operatorname{col}\left(x_{D}, x_{Q}\right) \in \mathbb{R}^{2}$. Then, we propose to use this transformation to map the zero component of steady-state $\Delta$-variables to constant quantities using

$$
x_{z D Q}^{\Delta}=T_{3 \omega}\left[\begin{array}{l}
x_{z}^{\Delta} \\
x_{z}^{\Delta \perp}
\end{array}\right] .
$$

Since an additional component has been introduced by the transformation, the $\Delta$-variables

$$
\begin{aligned}
v_{C z D Q}^{\Delta} & :=\operatorname{col}\left(v_{C z D}^{\Delta}, v_{C z Q}^{\Delta}\right) \in \mathbb{R}^{2}, \\
m_{z D Q}^{\Delta} & :=\operatorname{col}\left(m_{z D}^{\Delta}, m_{z Q}^{\Delta}\right) \in \mathbb{R}^{2}
\end{aligned}
$$

are further defined.

\section{Overall model with time-invariant solutions}

Before presenting the final MMC $d q z$ model with time invariant solutions, based on the transformations (3)-(5), an additional yet simple variables and parameters change is adopted. This slight modification, will ease the derivation of the system representation in port-Hamiltonian form that will be carried out in the next section. We then introduce the following variables

$$
\hat{m}_{z}^{\Sigma}:=2 m_{z}^{\Sigma}, \quad \hat{v}_{C z}^{\Sigma}:=2 v_{C z}^{\Sigma}, \quad \hat{i}_{z}^{\Sigma}:=2 i_{z}^{\Sigma}, \quad \hat{i}_{d q}^{\Delta}:=i_{d q}^{\Delta} / 2
$$

and the reparametrization:

\begin{tabular}{|c|c|c|c|}
\hline$\hat{L}_{\delta}:=8 L_{\delta}$ & $\hat{L}_{\sigma}:=8 L_{\sigma}$ & $\hat{L}_{\sigma z}:=4 L_{\sigma}$ & \\
\hline$\hat{R}_{\delta}:=8 R_{\delta}$ & $\hat{R}_{\sigma}:=8 R_{\sigma}$ & $\hat{R}_{\sigma z}:=4 R_{\sigma}$ & $\hat{G}_{d c}:=4 G_{d c} / 3$ \\
\hline & $\hat{C}_{\sigma}:=2 C_{\sigma}$ & $\hat{C}_{\sigma z}:=C_{\sigma}$ & $\hat{C}_{d c}:=4 C_{d c} / 3$ \\
\hline
\end{tabular}

Based on this change of coordinates, the MMC dynamics can be rewritten in compact form as

$$
P \dot{z}=A(m) z+B,
$$

with: state vector

$z:=\operatorname{col}\left(v_{C d q}^{\Sigma}, \hat{v}_{C z}^{\Sigma}, v_{C d q}^{\Delta}, v_{C z D Q}^{\Delta}, i_{C d q}^{\Sigma}, \hat{i}_{C z}^{\Sigma}, \hat{i}_{d q}^{\Delta}, v_{d c}\right) \in \mathbb{R}^{13}$,

control vector

$$
m:=\operatorname{col}\left(m_{d q}^{\Sigma}, \hat{m}_{z}^{\Sigma}, m_{d q}^{\Delta}, m_{z D Q}^{\Delta}\right) \in \mathbb{R}^{7},
$$

constant input vector

$$
B:=\operatorname{col}\left(0_{2}, 0,0_{2}, 0_{2}, 0_{2}, 0,-8 v_{G d q}^{\Delta},-\frac{4}{3} i_{d c, s}\right) \in \mathbb{R}^{13},
$$

and matrices:

$P=\operatorname{bdiag}\left\{\hat{C}_{\sigma} \mathbb{I}_{2}, \hat{C}_{\sigma z}, \hat{C}_{\sigma} \mathbb{I}_{4}, \hat{L}_{\sigma} \mathbb{I}_{2}, \hat{L}_{\sigma z}, \hat{L}_{\delta} \mathbb{I}_{2}, \hat{C}_{d c}\right\} \in \mathbb{R}^{13 \times 13}$. and $A(m) \in \mathbb{R}^{13 \times 13}$ defined as in (7).

\section{PI-PBC OF A Modular Multilevel Converter}

\section{A. PI-PBC of generalized power converters [11]}

The control methodology of interest consists in exploiting the results originally presented in [11], applicable to averaged models of switched power converters with steady-state timeinvariant solutions, which can be generalized in the following form, also called port-Hamiltonian representation:

$$
\dot{x}=\left(\mathcal{J}_{0}+\sum_{h=1}^{m} \mathcal{J}_{h} m_{h}-\mathcal{R}\right) \nabla \mathcal{H}(x)+E+G u,
$$

where $x \in \mathbb{R}^{n}$ is the state vector associated to the collection of charges $q_{j}=C_{j} v_{j}$ of the capacitors $C_{j}$ and the magnetic fluxes $\phi_{k}=L_{k} i_{k}$ of the inductors $L_{k} ; m_{h} \in \mathbb{R}^{m}$ are the duty ratios; $E \in \mathbb{R}^{n}$ is a vector of input sources, $u \in \mathbb{R}^{p}$ is the vector of interconnection variables; $\mathcal{J}_{i}=-\mathcal{J}_{i}^{\top}$ and $\mathcal{R}=\mathcal{R}^{\top} \geq 0$ are the $n$-dimensional square interconnection and dissipation matrices denoting respectively the internal configuration and the dissipation of the converter; $G \in \mathbb{R}^{n \times p}$ is an input matrix and

$$
\mathcal{H}(x)=\frac{1}{2} x^{\top} Q x=\frac{1}{2}\left(\sum_{j} \frac{q_{j}^{2}}{C_{j}}+\sum_{k} \frac{\phi_{k}^{2}}{L_{k}}\right)
$$

is the overall energy stored by the converter. As reported in [6], [7], [11], this representation allows to define the set of feasible steady-state for the system $x^{\star} \in \mathbb{R}^{n}$. Hence, for any feasible steady-state of interest $x^{\star} \in \mathcal{E}$, it is possible to define a simple PI controller of the form

$$
\begin{aligned}
m_{h} & =-K_{P h} y_{h}+K_{I h} z_{h}, \quad \dot{z}_{h}=-y_{h}, \\
y_{h} & =\nabla \mathcal{H}^{\top}\left(x^{\star}\right) \mathcal{J}_{h}^{\top} \nabla \mathcal{H}(x), \quad h=1 \ldots m,
\end{aligned}
$$

that guarantees that for all initial conditions $(x(0), z(0)) \in$ $\mathbb{R}^{n} \times \mathbb{R}^{m}$ the trajectories of the closed loop system are bounded and

$$
\lim _{t \rightarrow \infty} y(t)=0
$$

\section{B. Port-Hamiltonian modelling of the MMC}

For the application of this result to the case of the MMC it is sufficient to rewrite the system dynamics in the portHamiltonian form (8). For this purpose, we define the new state vector

$$
x=Q^{-1} z \in \mathbb{R}^{13}
$$

with $Q:=P^{-1} \in \mathbb{R}^{13 \times 13}$ and containing the collection of charges of the capacitors and the magnetic fluxes of the inductors, i.e.:

$$
x:=\operatorname{col}\left(q_{C d q}^{\Sigma}, \hat{q}_{C z}^{\Sigma}, q_{C d q}^{\Delta}, q_{C z D Q}^{\Delta}, \phi_{C d q}^{\Sigma}, \hat{\phi}_{C z}^{\Sigma}, \hat{\phi}_{d q}^{\Delta}, q_{d c}\right) \in \mathbb{R}^{13} .
$$

Furthermore, let the matrix $A(m)$ be decomposed in a symmetric positive $\mathcal{R}=\mathcal{R}^{\top} \geq 0$ and skew-symmetric part $\mathcal{J}=-\mathcal{J}^{\top}$, so that (6) can be rewritten as

$$
\dot{x}=(\mathcal{J}(m)-\mathcal{R}) \nabla \mathcal{H}+E+G u, \quad \mathcal{H}(x):=\frac{1}{2} x^{\top} Q x,
$$

where we have further introduced the decomposition $B=E+$ $G u$, with $u=i_{d c, s} \in \mathbb{R}^{3}$ and $E, G \in \mathbb{R}^{13}$ defined in the Appendix. Moreover it is easy to see that the control variables appears linearly in (7), so that it is possible to write

$$
\mathcal{J}(m):=\mathcal{J}_{0}+\sum_{h=1}^{7} \mathcal{J}_{h} m_{h}
$$

for some appropriate matrices $\mathcal{J}_{0}, \mathcal{J}_{h} \in \mathbb{R}^{13 \times 13}$. For a detailed expression of these matrices the reader is referred to the 


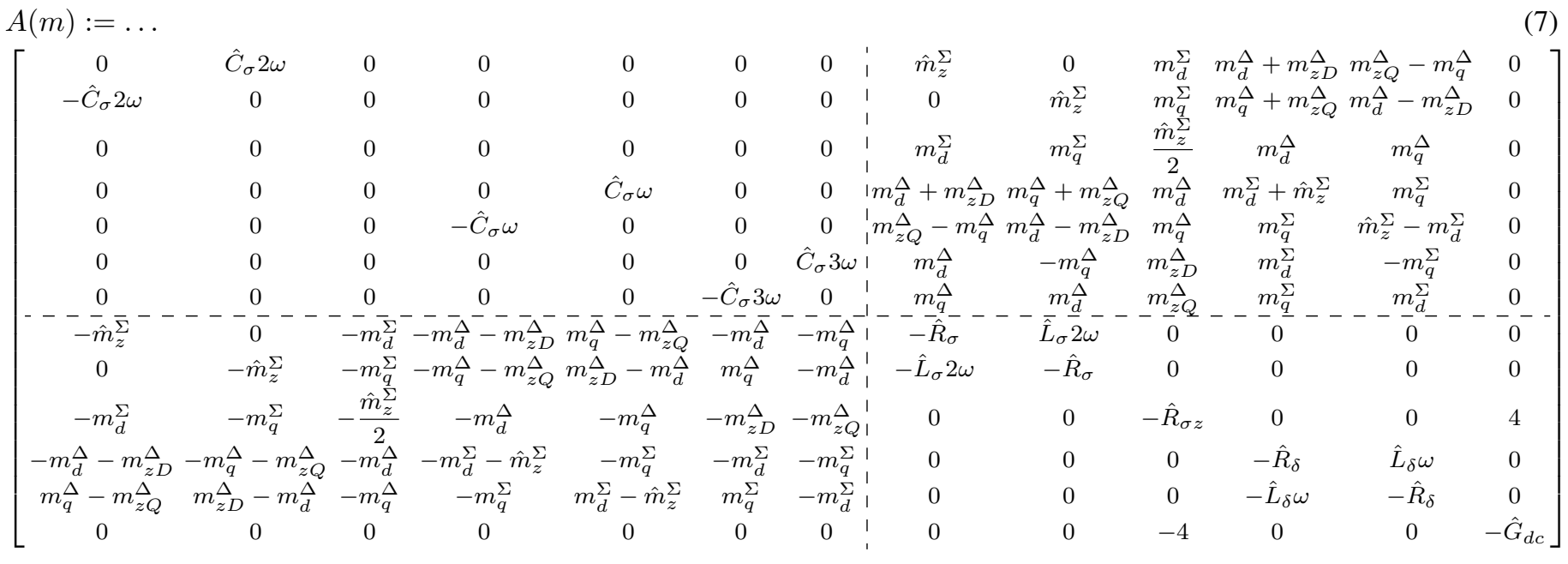

Appendix. Since the obtained model of the MMC matches with the port-Hamiltonian system (8), the same result reported in [11] can be applied. In particular, the passive output can be computed according to (9). Since the MMC has seven control inputs, we have

$$
\begin{aligned}
& y_{1}=\left[\hat{i}_{z}^{\Sigma \star} v_{C d}^{\Sigma} \hat{i}_{z}^{\Sigma} v_{C d}^{\Sigma \star}\right]+\left[i_{d}^{\Sigma \star} \hat{v}_{C z^{\Sigma}}^{\Sigma}-i_{d}^{\Sigma} \hat{v}_{C z}^{\Sigma \star}\right]+\left[i_{d}^{\Delta \star}\left(v_{C d}^{\Delta}+v_{C z D}^{\Delta}\right)\right. \\
& \left.-\left(v_{C d}^{\Delta \star}+v_{C z D}^{\Delta \star}\right) i_{d}^{\Delta}\right]+\left[i_{q}^{\Delta \star}\left(v_{C z Q^{-}}^{\Delta} v_{C q}^{\Delta}\right)-\left(v_{C z Q^{-}}^{\Delta \star} v_{C q}^{\Delta \star}\right) i_{q}^{\Delta}\right] \\
& y_{2}=\left[\hat{i}_{z}^{\Sigma \star} v_{C q}^{\Sigma}-v_{C q}^{\Sigma \star} \hat{i}_{z}^{\Sigma}\right]^{-}+\left[i_{q}^{\Sigma \star} \hat{v}_{C z}^{\Sigma}-\hat{v}_{C z}^{\Sigma \star} i_{q}^{\Sigma}\right]+\left[i_{q}^{\star \star}{ }^{-}\left(v_{C d}^{\Delta}-v_{C z D}^{\Delta}\right)\right.
\end{aligned}
$$

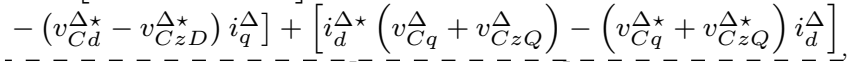

$$
\begin{aligned}
& y_{3}=\left[i_{d}^{\Sigma \star} v_{C d}^{\Sigma}-v_{C d}^{\Sigma \star} i_{d}^{\Sigma}\right]+\left[i_{q}^{\Sigma \star} v_{C q}^{\Sigma}-v_{C q}^{\Sigma \star} i_{q}^{\Sigma}\right]+\left[i_{d}^{\bar{\Delta} \star} v_{C d}^{\bar{\Delta}}-v_{C}^{\bar{\Delta}} i_{d}^{\Delta}\right] \\
& +\frac{1}{2}\left[\hat{i}_{z}^{\Sigma \star} \hat{v}_{C z}^{\Sigma}-\hat{v}_{C z}^{\Sigma \star} \hat{i}_{z}^{\Sigma}\right]+\left[i_{q}^{\Delta \star} v_{C q}^{\Delta}-v_{C q}^{\Delta \star} i_{q}^{\Delta}\right]
\end{aligned}
$$

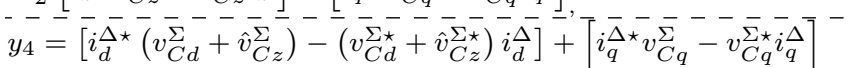

$$
\begin{aligned}
& +\left[i_{d}^{\Sigma \star}\left(v_{C d}^{\Delta}+v_{C d}^{\Delta}\right)-\left(v_{C d}^{\Delta \star}+v_{C}^{\Delta \star}\right) i_{d}^{\Sigma}\right]+\left[\hat{i}_{z}^{\Sigma \star} v_{C d}^{\Delta}-v_{C d}^{\Delta} \hat{i}_{z}^{\Sigma}\right] \\
& +\left[i_{q}^{\Sigma \star}\left(v_{\vec{C} q}^{\Delta}+v_{C z Q}^{\Delta}\right)-\left(v_{C_{q}^{\star}}^{\Delta \star}+v_{C z Q}^{\Delta \star}\right) i_{q}^{\Sigma}\right] \\
& y_{5}=\left[i_{q}^{\Delta \star}\left(\hat{v}_{C z}^{\Sigma}-v_{C d}^{\Sigma}\right)-\left(\hat{v}_{C z}^{\Sigma \star}-v_{C d}^{\Sigma_{d}}\right) \overline{\left.i_{q}^{\Delta}\right]}+\left[i_{d}^{\bar{\Delta} \star} \bar{v}_{C q}^{\bar{\Sigma}}-\bar{v}_{C q}^{\Sigma_{q}} \bar{i}_{d}^{\Delta}\right]\right. \\
& +\left[i_{q}^{\Sigma \star}\left(v_{C d}^{\Delta}-v_{C z D}^{\Delta}\right)-\left(v_{C d}^{\Delta \star}-v_{C z D}^{\Delta \star}\right) i_{q}^{\Sigma}\right]+\left[i_{d}^{\Sigma \star}\left(v_{C z Q}^{\Delta}-v_{C q}^{\Delta}\right)\right. \\
& \left.-\left(v_{C z Q}^{\Delta \star}-v_{C q}^{\Delta \star}\right) i_{d}^{\Sigma}\right]+\left[\hat{i}_{z}^{\Sigma \star} v_{C q}^{\Delta}-v_{C q}^{\Delta \star} \hat{i}_{z}^{\Sigma}\right] \\
& y_{6}=\left[i_{d}^{\Delta \star} v_{C d}^{\Sigma}-v_{C d}^{\Sigma \star} i_{d}^{\Delta}\right]-\left[i_{q}^{\Delta \star} v_{C q}^{\Sigma}-v_{C q}^{\Sigma \star} i_{q}^{\Delta}\right]+\left[i_{d}^{\Sigma \star} v_{C d}^{\Delta}-v_{C d}^{\Delta} i_{d}^{\Sigma}\right] \\
& -\left[i_{q}^{\Sigma \star} v_{C q}^{\Delta}-v_{C q}^{\Delta \star} i_{q}^{\Sigma}\right]+\left[\hat{i}_{z}^{\Sigma \star} v_{C z D}^{\Delta}-v_{C z D}^{\Delta \star} \hat{i}_{z}^{\Sigma}\right] \\
& y_{7}=\left[i_{q}^{\Delta \star} v_{C d}^{\Sigma}-v_{C d}^{\Sigma \star} i_{q}^{\Delta}\right]+\left[i_{d}^{\Delta \star} v_{C q}^{\Sigma}-v_{C q}^{\Sigma \star} i_{d}^{\Delta}\right]+\left[i_{q}^{\Sigma \star} v_{C d}^{\Delta}-v_{C d}^{\Delta} i_{q}^{\Sigma}\right] \\
& +\left[i_{d}^{\Sigma \star} v_{C q}^{\Delta}-v_{C q}^{\Delta \star} i_{d}^{\Sigma}\right]+\left[\hat{i}_{z}^{\Sigma \star} v_{C}^{\Delta} z Q-v_{C z Q}^{\Delta \star} \hat{i}_{z}^{\Sigma}\right] \text {. }
\end{aligned}
$$

\section{MT-HVDC BASED ON MMCS IN RADIAL CONFIGURATION}

We now extend the result obtained in the previous section to the case of a generalized MT-HVDC system based on MMCs, in radial configuration. An example of such a configuration is illustrated in Fig. 3. For the derivation of the interconnected model, we assume that the system is characterized by c MMCs and that the dc transmission lines can be described by standard single-cell $\pi$-models, converging at a point of common connection (PCC). The procedure for deriving the controller mimics the procedure adopted for the single-terminal case, i.e. the system is first represented using a port-Hamiltonian description, that allows to determine a passive output. Then, a simple PI control, based on this output, is designed, in order to ensure global asymptotic stability of the closed-loop system.

\section{A. Port-Hamiltonian representation of the transmission lines}

As illustrated in Fig. 3, in a radial configuration each MMC associates a different $\pi$-section. Hence, at any point of connection between and MMC and the correspondent line, two capacitors result connected in parallel [6]. For simplicity we assume then to replace such capacitors with an equivalent capacitor $C_{d c, i} \in \mathbb{R}_{+}$, which is considered as a part of the MMC. Similarly, c capacitors are connected in parallel at the PCC, which can be described by an equivalent capacitor $C_{0} \in \mathbb{R}_{+}$. Based on these considerations, the dynamics of the lines are then described by the following system

$$
\left[\begin{array}{l}
L_{T} \dot{i}_{T} \\
C_{0} \dot{v}_{0}
\end{array}\right]=\left[\begin{array}{cc}
-R_{T} & -1 \\
1^{\top} & 0
\end{array}\right]\left[\begin{array}{l}
i_{T} \\
v_{0}
\end{array}\right]+\left[\begin{array}{c}
\mathbb{I}_{\mathrm{c}} \\
0
\end{array}\right] V_{T}
$$

with $i_{T} \in R^{\mathrm{c}}$ the vector of lines currents, $V_{T} \in \mathbb{R}^{\mathrm{c}}$ the vector of input voltages of the lines, $v_{0} \in \mathbb{R}$ the voltage at the PCC, $R_{T} \in \mathbb{R}^{\mathrm{c} \times \mathrm{c}}$ a diagonal matrix containing the inductor resistances. It is easy to show that the dynamics (11) admit the following port-Hamiltonian representation

$$
\begin{aligned}
& \dot{x}_{\text {net }}=\left(\mathcal{J}_{\text {net }}-\mathcal{R}_{\text {net }}\right) \nabla \mathcal{H}_{\text {net }}+G_{\text {net }} u_{\text {net }} \\
& y_{\text {net }}=G_{\text {net }}^{\top} \nabla \mathcal{H}_{\text {net }}
\end{aligned}
$$

with state vector $x_{\text {net }}:=\operatorname{col}\left(\psi_{T}, q_{0}\right) \in \mathbb{R}^{\mathrm{c}+1}$, where $\psi_{T} \in$ $\mathbb{R}^{c}$ corresponds to the collection of lines magnetic fluxes and $q_{0}$ to the charge at the PCC; interconnection variables $u_{\text {net }}:=V_{\text {net }} \in \mathbb{R}^{\mathrm{c}}$ and $y_{\text {net }}:=I_{\text {net }} \in \mathbb{R}^{\mathrm{c}}$, where $V_{\text {net }}, I_{\text {net }}$ correspond respectively to the collection of input voltages and lines currents; interconnection, dissipation and input matrices

$$
\mathcal{J}_{\text {net }}:=\left[\begin{array}{cc}
0 & -1 \\
1^{\top} & 0
\end{array}\right], \quad \mathcal{R}_{\text {net }}=\left[\begin{array}{cc}
-R_{T} & 0 \\
0 & 0
\end{array}\right], \quad G_{\text {net }}:=\left[\begin{array}{c}
\mathbb{I}_{\mathrm{c}} \\
0_{\mathrm{c}}^{\top}
\end{array}\right]
$$


and the energy function

$$
\mathcal{H}_{\mathrm{net}}\left(\psi_{T}, q_{0}\right):=\frac{1}{2} \psi_{T}^{\top} L_{T}^{-1} \psi_{T}+\frac{1}{2} \frac{q_{0}^{2}}{C_{0}} .
$$

\section{B. Aggregated port-Hamiltonian representation of the MMCs}

The port Hamiltonian representation of a single-terminal MMC station has been identified in Section III. Since we are considering c MMCs in the multi-terminal configuration, it is convenient to aggregate all the c port-Hamiltonian models of the MMCs, thus resulting in

$$
\begin{aligned}
\dot{x}_{\mathrm{c}} & =\left(\mathcal{J}_{\mathrm{c}}\left(m_{\mathrm{c}}\right)-\mathcal{R}_{\mathrm{c}}\right) \nabla \mathcal{H}_{\mathrm{c}}+E_{\mathrm{c}}+G_{\mathrm{c}} u_{\mathrm{c}} \\
y_{\mathrm{c}} & =G_{\mathrm{c}}^{\top} \nabla \mathcal{H}_{\mathrm{c}}
\end{aligned}
$$

with state vector $x_{\mathrm{c}}:=\operatorname{col}\left(x_{i}\right) \in \mathbb{R}^{c n}$, interconnection variables $u_{\mathrm{c}}:=I_{\mathrm{c}} \in \mathbb{R}^{\mathrm{c}}$ and $y_{\mathrm{c}}:=V_{\mathrm{c}} \in \mathbb{R}^{\mathrm{c}}$, where $I_{\mathrm{c}}, V_{\mathrm{c}}$ correspond respectively to the collection of input currents and voltages of the MMCs; interconnection, dissipation matrices

$\mathcal{J}_{\mathrm{c}}:=\operatorname{bdiag}\left\{\mathcal{J}_{i}\right\} \in \mathbb{R}^{13 c \times 13 c}, \mathcal{R}_{\mathrm{c}}:=\operatorname{bdiag}\left\{\mathcal{R}_{i}\right\} \in \mathbb{R}^{13 c \times 13 c}$, sources vector and input matrix

$$
E:-\operatorname{col}\left(E_{i}\right) \in \mathbb{R}^{13 c}, \quad G_{\mathrm{c}}:=\operatorname{bdiag}\left\{G_{i}\right\} \in \mathbb{R}^{13 c \times c}
$$

and the energy function

$$
\mathcal{H}_{\mathrm{c}}(x):=\sum_{i=1}^{\mathrm{c}} \mathcal{H}_{i}\left(x_{i}\right)
$$

\section{Port-Hamiltonian representation of the MT-HVDC system}

To obtain the full interconnected model of the MT-HVDC system, it is now sufficient to interconnect (12), (13) using Kirkchhoff's, power preserving, laws, which can be written in the following compact form

$$
\left[\begin{array}{c}
I_{\mathrm{c}} \\
V_{\text {net }}
\end{array}\right]=\frac{3}{4}\left[\begin{array}{rr}
0 & \mathbb{I}_{\mathrm{c}} \\
-\mathbb{I}_{\mathrm{c}} & 0
\end{array}\right]\left[\begin{array}{c}
V_{\mathrm{c}} \\
I_{\text {net }}
\end{array}\right]
$$

where the scaling factor $3 / 4$ has been further introduced to preserve skew-symmery of the interconnected system. We then introduce the following reparametrization: $\hat{L}_{T}:=4 L_{T} / 3, \hat{C}_{0}:=$ $4 C_{0} / 3$, such that we can define $\hat{x}_{\text {net }}:=\operatorname{col}\left(\hat{L}_{T} i_{T}, \hat{C}_{0} v_{0}\right)=$ $\operatorname{col}\left(\hat{\phi}_{T}, \hat{q}_{0}\right)$. Combining (14) with (12) and (13), we then obtain the following, overall port-Hamiltonian representation of the MT-HVDC system

$$
\dot{x}_{\text {tot }}=\left(\mathcal{J}_{\text {tot }}\left(m_{\mathrm{c}}\right)-\mathcal{R}_{\text {tot }}\right) \nabla \mathcal{H}_{\text {tot }}+E_{\text {tot }}
$$

with state vector $x_{\text {tot }}:=\operatorname{col}\left(x_{\mathrm{c}}, \hat{x}_{\text {net }}\right) \in \mathbb{R}^{14 \mathrm{c}+1}$, interconnection, dissipation matrices and source vector

$$
\begin{gathered}
\mathcal{J}_{\text {tot }}\left(m_{\mathrm{c}}\right):=\left[\begin{array}{cc}
\mathcal{J}_{\mathrm{c}}\left(m_{\mathrm{c}}\right) & G_{\mathrm{c}} G_{\text {net }}^{\top} \\
-G_{\text {net }} G_{\mathrm{c}}^{\top} & \frac{4}{3} \mathcal{J}_{\text {net }}
\end{array}\right], \\
\mathcal{R}_{\text {tot }}:=\operatorname{bdiag}\left\{\mathcal{R}_{\mathrm{c}}, \frac{4}{3} \mathcal{R}_{T}\right\}, \quad E_{\text {tot }}:=\operatorname{col}\left(E, 0_{\mathrm{c}+1}\right)
\end{gathered}
$$

and energy function $\mathcal{H}_{\text {tot }}=\mathcal{H}_{\mathrm{c}}+\mathcal{H}_{\text {net }}$.

It is now easy to see that the obtained representation is again equivalent to the port-Hamiltonian representation of generalized power converter given by (8), thus allowing the application of the same results. Interestingly, the same output (10) is obtained for each MMC, which implies that the same decentralized PI-PBC controllers ensure global asymptotic stability of the interconnected system.

\section{Simulation Results}

The proposed decentralized passivity-based PI is tested on a MT-HVDC system based on MMCs with three terminals in radial topology, i.e. $\mathrm{c}=3 \mathrm{MMCs}$, see Fig. 3. Each MMC has an apparent nominal power of $S n=1200 M V A$, and a nominal voltage of $380 \mathrm{kV}$. Furthermore each MMC is characterized by the following parameters: equivalent arm capacitance $C_{\sigma}=21.162 \mu \mathrm{F}$; equivalent arm inductance $L_{\sigma}=30 \mathrm{mH}$ and equivalent ac inductance $L_{\delta}=78 \mathrm{mH}$. For the excitation of the MT-HVDC dynamics, an active current reference step change is given to MMC\#1 at $t=5 \mathrm{~ms}$ from $0.85 \mathrm{pu}$ to $-0.85 \mathrm{pu}$. Moreover, the active current of MMC \#3 is changed from $0.45 \mathrm{pu}$ to -0.45 at $8.5 \mathrm{~ms}$. This is illustrated in Fig. 4, where in addition to the expected behaviour of MMC $\# 1$ and \#3, it can be seen that MMC \#2 acts by compensating the power unbalance. The zero-sequence of the circulating current - or equivalently one third of the dc terminal currentis illustrated in Fig. 5. Finally, the dc terminal voltages depicted in Fig. 6 as well as the aggregated voltage of all the MMC internal capacitors - see Fig. 7-are regulated to their desired values despite the perturbation. The control method under study, as expected, ensures global asymptotic stability in a decentralized manner.

Unfortunately, the PI-PBC gives below par performance for this application. Similar to the 2L-VSC case reported in [6], [7], the convergence rate of the system is extremely slow. To avoid these slow dynamics in the results here presented, we have added to the PI control its expected value at the desired equilibrium; i.e., $u^{\star}$. Nonetheless, this solution is but a partial fix as it seems to cause undesirable high voltage transients, as shown in Fig. 6. Therefore, future efforts will be directed at improving the performance of the PI-PBC applied to MMCbased MT-HVDC systems.

\section{CONCLUSION}

We have applied a passivity based PI controller (PI-PBC) to the Modular Multilevel Converter (MMC) under both, singleterminal HVDC configuration, as well as for the radial multiterminal (MT) case.

The procedure takes as a starting point a convenient reformulation of the averaged MMC single-terminal model in $d q z$ coordinates with fixed equilibrium point instead of the standard formulation based on its natural time-periodic coordinates. Furthermore, it is shown that this reformulation admits a Port-Hamiltonian $(\mathrm{pH})$ representation suitable for the PI-PBC method. After identifying the MMC interconnection, damping and input matrices, as well as its energy function or Hamiltonian, it is then straightforward to calculate the socalled passive output of this converter, upon which a standard PI around it renders the system globally asymptotically stable.

In addition, the MT-HVDC case in radial configuration was investigated. Since standard $\pi$-models of radially connected transmission lines admit a $\mathrm{pH}$ representation, they have been combined with the $\mathrm{pH}$ model of the $\mathrm{c} \mathrm{MMCs}$ to form the model of the full interconnected system, which is itself $\mathrm{pH}$. Surprisingly enough, the passive output of the full MT-HVDC system 


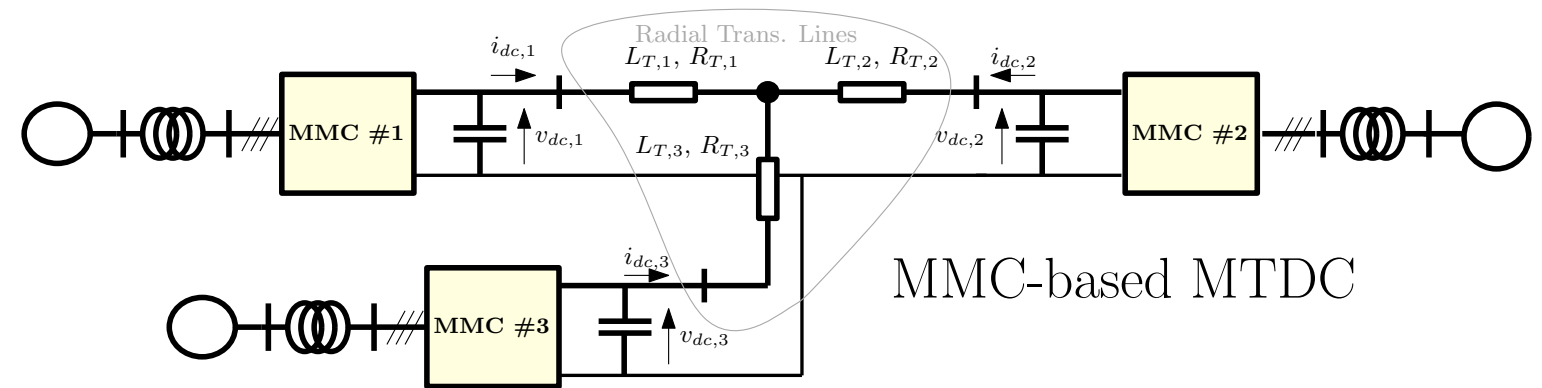

Figure 3: Multi-Terminal HVDC configuration under consideration

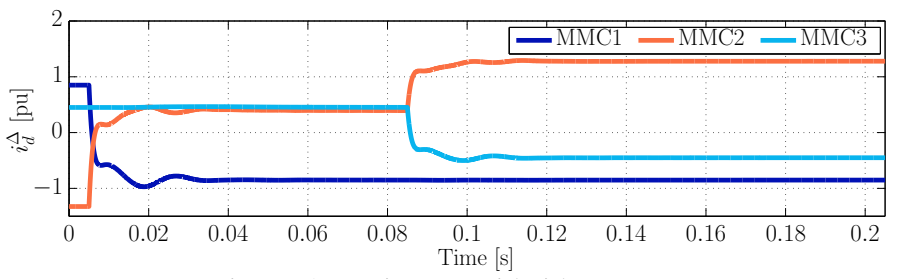

Figure 4: Active ac grid-side current

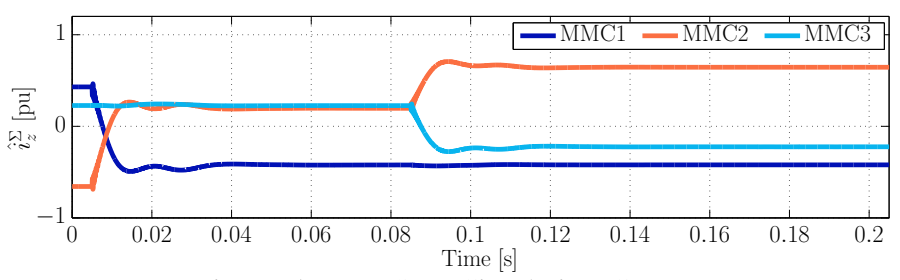

Figure 5: Zero-Seq. Circulating Current

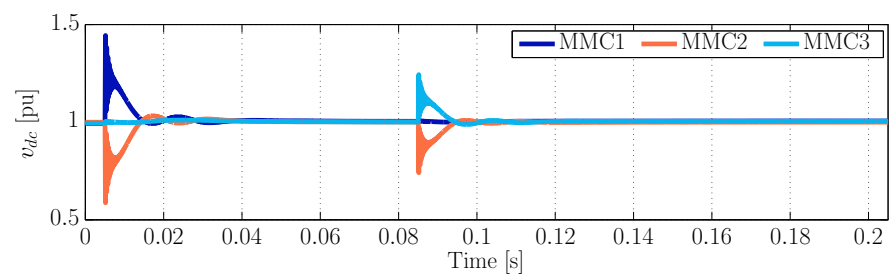

Figure 6: Voltage between de terminals

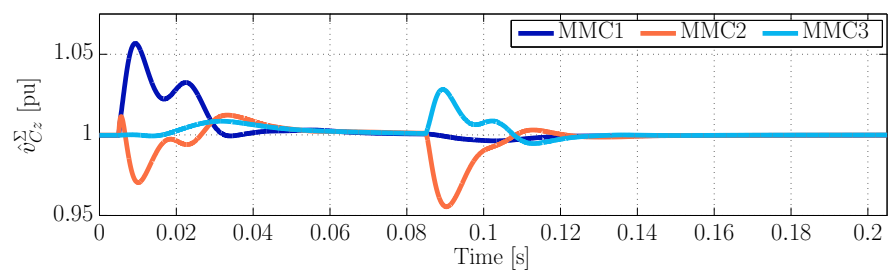

Figure 7: Zero-Seq. of Capacitor Voltage Sum

is the union of the passive outputs of each of the individual MMCs, giving this controller its decentralized feature, while achieving global asymptotic stability for the interconnected system.

\section{APPENDIX}

The dissipation matrix $\mathcal{R}$ of the MMC can be expressed as

$$
\mathcal{R}:=\operatorname{diag}\left(0_{7}^{\top}, \hat{R}_{\sigma}, \hat{R}_{\sigma}, \hat{R}_{\sigma z}, \hat{R}_{\delta}, \hat{R}_{\delta}, \hat{G}_{d c}\right) \in \mathbb{R}^{13 \times 13}
$$

and the interconnection matrix $\mathcal{J}_{0}$ as:

$$
\mathcal{J}_{0}=\left[\begin{array}{c:c}
\overline{\mathcal{J}_{0}} & 0_{9} \\
\hdashline 0_{9}^{\top}-\overline{4} \overline{0}_{2}^{\top} & 0_{2}
\end{array}\right] \in \mathbb{R}^{13 \times 13}
$$

with $\overline{\mathcal{J}_{0}} \in \mathbb{R}^{12 \times 12}$ defined as

$\overline{\mathcal{J}_{0}}:=\operatorname{bdiag}\left(\mathbb{J}_{2} \hat{C}_{\sigma} 2 \omega, 0, \mathbb{J}_{2} \hat{C}_{\sigma} \omega, \mathbb{J}_{2} \hat{C}_{\sigma} 3 \omega, \mathbb{J}_{2} \hat{L}_{\sigma} 2 \omega, 0, \mathbb{J}_{2} \hat{L}_{\delta} \omega\right)$,

with $\mathbb{J}_{2}:=\left[\begin{array}{rr}0 & 1 \\ -1 & 0\end{array}\right] \in \mathbb{R}^{2 \times 2}$.

Furthermore, the MMC has 7 control modulated interconnection matrices $\mathcal{J}_{h} \in \mathbb{R}^{13 \times 13}$; which can all be directly identified from (7), and are given in the following lines. Each of the 7 interconnection matrices can be written as a function of 4 sub-matrices with the form

$$
\mathcal{J}_{h}:=\left[\begin{array}{c:c}
{\overline{\mathcal{J}_{h}}}^{\top}{ }^{\prime} & 0_{12} \\
-\overline{\mathcal{J}}_{h}^{\top} & { }^{\top} \\
\hdashline 0_{12}^{\top}- & 0
\end{array}\right] \in \mathbb{R}^{13 \times 13},
$$

with $\overline{\mathcal{J}_{h}} \in \mathbb{R}^{7 \times 5}$ defined as $\overline{\mathcal{J}_{h}}:=\left[\begin{array}{ll}\overline{A_{h}} & \overline{B_{h}} \\ \overline{C_{h}} & \overline{D_{h}}\end{array}\right]$ for some $\overline{A_{h}} \in \mathbb{R}^{3 \times 3}, \overline{B_{h}} \in \mathbb{R}^{3 \times 2}, \overline{C_{h}} \in \mathbb{R}^{4 \times 3}$ and $\overline{D_{h}} \in \mathbb{R}^{4 \times 2}$. Of these sub-matrices, those which are non-zero are given below for $h=1 \ldots 7$.

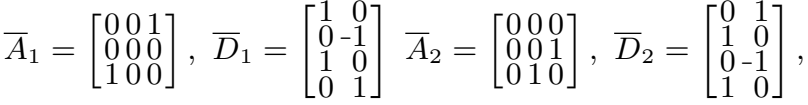

$$
\begin{aligned}
& \bar{A}_{3}=\left[\begin{array}{lll}
1 & 0 & 0 \\
0 & 1 & 0 \\
0 & 0 & \frac{1}{2}
\end{array}\right], \bar{D}_{3}=\left[\begin{array}{ll}
1 & 0 \\
0 & 1 \\
0 & 0 \\
0 & 0
\end{array}\right], \bar{B}_{4}=\left[\begin{array}{ll}
1 & 0 \\
0 & 1 \\
1 & 0
\end{array}\right], \bar{C}_{4}=\left[\begin{array}{lll}
1 & 0 & 1 \\
1 & 0 & 0 \\
1 & 0 & 0 \\
0 & 1 & 0
\end{array}\right], \\
& \bar{B}_{5}=\left[\begin{array}{rr}
0 & -1 \\
1 & 0 \\
0 & 1
\end{array}\right], \bar{C}_{5}=\left[\begin{array}{rrr}
0 & 1 & 0 \\
-1 & 0 & 1 \\
0 & -1 & 0 \\
1 & 0 & 0
\end{array}\right], \bar{B}_{6}=\left[\begin{array}{rr}
1 & 0 \\
0 & -1 \\
0 & 0
\end{array}\right], \bar{C}_{6}=\left[\begin{array}{rrr}
1 & 0 & 0 \\
0 & -1 & 0 \\
0 & 0 & 1 \\
0 & 0 & 0
\end{array}\right] \text {, } \\
& \bar{B}_{7}=\left[\begin{array}{ll}
0 & 1 \\
1 & 0 \\
0 & 0
\end{array}\right], \bar{C}_{7}=\left[\begin{array}{lll}
0 & 1 & 0 \\
1 & 0 & 0 \\
0 & 0 & 0 \\
0 & 0 & 1
\end{array}\right]
\end{aligned}
$$

Finally, the input and the interconnection vectors of the MMC $E \in \mathbb{R}^{13}$ and $G \in \mathbb{R}^{13}$, can be directly identified from the vector $B$ in (6), noting that $B=E+G u$. Thus, for the selected input $u=i_{d c, s}$, they can be respectively defined as

$$
E:=\operatorname{col}\left(0_{10},-8 v_{G d q}^{\Delta}, 0\right) ; \quad G:=\operatorname{col}\left(0_{12},-\frac{4}{3}\right) .
$$




\section{ACKNOWLEDGMENT}

This work was supported by the Norwegian Research Council and DNV-GL through the NFR contract n. 250493within the "Integrated Design and Control of Offshore HVDC Networks (IDeCON)" project-and by the European Commission through the AMAROUT-II Marie Curie Researcher Mobility Action under Grant FP7-PEOPLE-2013-COFUND.

\section{REFERENCES}

[1] "Bestpaths eu project," http://bestpaths-project.eu/, accessed: 2017-0626.

[2] S. Chatzivasileiadis, D. Ernst, and G. Andersson, Global Power Grids for Harnessing World Renewable Energy. Amsterdam: Elsevier, 2014.

[3] D. V. Hertem, O. Gomis-Bellmunt, and J. Liang, IEEE Press Series on Power Engineering. WileyIEEE Press, 2016, pp. 528-. [Online]. Available: http://ieeexplore.iee. $.0 \mathrm{~g} / \mathrm{xpl} /$ articleDetails.jsp?arnumber $=7435160$

[4] J. Beerten, S. D'Arco, and J. A. Suul, "Identification and small-signal analysis of interaction modes in vsc mtdc systems," IEEE Transactions on Power Delivery, vol. 31, no. 2, pp. 888-897, April 2016.

[5] H. K. Khalil and J. Grizzle, Nonlinear systems. Prentice hall New Jersey, 1996, vol. 3.

[6] D. Zonetti, R. Ortega, and A. Benchaib, "Modeling and control of HVDC transmission systems from theory to practice and back," Control Engineering Practice, vol. 45, pp. 133 - 146, 2015. [Online]. Available: http://www.sciencedirect.com/science/article/pii/S096706611530023X

[7] _ " "A globally asymptotically stable decentralized pi controller for multi-terminal high-voltage dc transmission systems," in 2014 European Control Conference (ECC), June 2014, pp. 1397-1403.

[8] A. Lesnicar and R. Marquardt, "An innovative modular multilevel converter topology suitable for a wide power range," in Power Tech Conference Proceedings, 2003 IEEE Bologna, vol. 3, June 2003, pp. 6 pp. Vol.3-

[9] A. Antonopoulos, L. Angquist, and H. P. Nee, "On dynamics and voltage control of the modular multilevel converter," in 2009 13th European Conference on Power Electronics and Applications, Sept 2009, pp. 1-10.

[10] L. Harnefors, A. Antonopoulos, S. Norrga, L. Angquist, and H.-P. Nee, "Dynamic analysis of modular multilevel converters," Industrial Electronics, IEEE Transactions on, vol. 60, no. 7, pp. 2526-2537, July 2013
[11] M. Hernandez-Gomez, R. Ortega, F. Lamnabhi-Lagarrigue, and G. Escobar, "Adaptive pi stabilization of switched power converters," IEEE Transactions on Control Systems Technology, vol. 18, no. 3, pp. 688698, May 2010.

[12] G. Bergna, J. A. Suul, and S. D'Arco, "State-space modelling of modular multilevel converters for constant variables in steady-state," in 2016 IEEE 17th Workshop on Control and Modeling for Power Electronics (COMPEL), June 2016, pp. 1-9.

[13] G. Bergna-Diaz, J. Freytes, X. Guillaud, S. D’Arco, and J. A. Suul, "Generalized voltage-based state-space modelling of modular multilevel converters with constant equilibrium in steady-state," 2017, cite arxiv:1706.04959. [Online]. Available: http://arxiv.org/abs/1706.04959

[14] G. Bergna-Diaz, S. Sanchez, and E. Tedeschi, "Port-hamiltonian modelling of modular multilevel converters with fixed equilibrium point," in 2017 Twelfth International Conference on Ecological Vehicles and Renewable Energies (EVER), April 2017, pp. 1-12.

[15] G. Escobar, A. J. Van Der Schaft, and R. Ortega, "A hamiltonian viewpoint in the modeling of switching power converters," Automatica, vol. 35, no. 3, pp. 445-452, 1999.

[16] S. Rohner, J. Weber, and S. Bernet, "Continuous model of modular multilevel converter with experimental verification," in 2011 IEEE Energy Conversion Congress and Exposition, Sept 2011, pp. 40214028.

[17] A. Christe and D. Duji, "State-space modeling of modular multilevel converters including line frequency transformer," in Power Electronics and Applications (EPE'15 ECCE-Europe), 2015 17th European Conference on, Sept 2015, pp. 1-10.

[18] H. Saad, S. Dennetière, J. Mahseredjian, P. Delarue, X. Guillaud, J. Peralta, and S. Nguefeu, "Modular multilevel converter models for electromagnetic transients," IEEE Transactions on Power Delivery, vol. 29, no. 3, pp. 1481-1489, June 2014.

[19] A. Antonopoulos, L. Angquist, and H. P. Nee, "On dynamics and voltage control of the modular multilevel converter," in Power Electronics and Applications, 2009. EPE '09. 13th European Conference on, Sept 2009, pp. 1-10.

[20] Q. Tu, Z. Xu, and L. Xu, "Reduced switching-frequency modulation and circulating current suppression for modular multilevel converters," in Transmission and Distribution Conference and Exposition (T D), 2012 IEEE PES, May 2012, pp. 1-1.

[21] R. Teodorescu, M. Liserre, and P. Rodríguez, Grid converters for photovoltaic and wind power systems. IEEE/John Wiley \& Sons, 2011, vol. 29. 